\title{
Psychological Healthcare Burden Lessens After Hip Arthroscopy for Those With Comorbid Depression or Anxiety
}

\author{
Anthony J. Zacharias, M.D., Nicole G. Lemaster, Ph.D., A.T.C., Gregory S. Hawk, M.S., \\ Stephen T. Duncan, M.D., Katherine L. Thompson, Ph.D., Kate N. Jochimsen, Ph.D., \\ Austin V. Stone, M.D., Ph.D., and Cale A. Jacobs, Ph.D.
}

\begin{abstract}
Purpose: In this study, we investigated whether patients undergoing arthroscopic treatment of femoral acetabular impingement syndrome (FAIS) seek health care for treatment of comorbid depression and anxiety in the year following hip arthroscopy. Methods: Using the Truven Health Marketscan database, FAIS patients who underwent hip arthroscopy between January 2009 and December 2016 were identified. Claims related to depression or anxiety filed during the year before surgery were required for inclusion. Using claims for pharmaceutical and psychological therapy treatments for mental health disorders, four groups of patients were analyzed on the basis of preoperative anxiety/depression treatment: medication only, therapy only, medication + therapy, and no treatment. Number of opioid pain prescriptions within 180 days prior to surgery and $>90$ days after hip arthroscopy were also compared. Results: Depression and anxiety claims were identified in 5,208/14,830 (35.1\%) patients. Preoperative treatment for depression and anxiety included medication only $(n=648,12.4 \%)$, therapy only $(n=899,17.3 \%)$, medication + therapy $(n=252,4.8 \%)$, and no treatment $(n=$ $3,409,65.5 \%)$. Of the 900 patients who filled an anxiety/depression-related prescription prior to surgery, $422(46.9 \%)$ patients did not fill a similar prescription in the postoperative year. Of the 1,151 patients receiving anxiety/depressionrelated therapy prior to surgery, $549(47.7 \%)$ did not receive therapy in the postoperative year. Preoperative opioid prescriptions were filled for 393 patients $(60.6 \%)$ in medication-only group, 275 (30.6\%) in therapy-only group, 156 $(61.9 \%)$ in medication + therapy group, and $1,059(31.1 \%)$ in the group receiving no treatment. Opioid prescriptions $>90$ days postoperatively were filled for $330(50.9 \%), 225(25.0 \%), 120(47.6 \%)$, and $861(25.3 \%)$ patients, respectively. Conclusion: Hip arthroscopy for FAIS is associated with a decreased postoperative use of health care resources for the treatment of depression and anxiety. Clinicians should also be aware of the potential interplay between preoperative psychotropic medication use and prolonged opioid use when counseling patients. Level of Evidence: IV, therapeutic case series
\end{abstract}

\section{Introduction}

$\mathbf{T}$ he 2019 National Survey of Drug Use and Health showed $15.2 \%$ of individuals aged $18-25$ and $8.9 \%$

From the Department of Orthopaedic Surgery $\theta$ Sports Medicine, University of Kentucky, Lexington, Kentucky, U.S.A. (A.J.Z., S.T.D., C.A.J.); Division of Sports Medicine, Department of Orthopaedic Surgery $\theta$ Sports Medicine, University of Kentucky, Lexington, Kentucky, U.S.A. (A.V.S.); Department of Rehabilitation Science, University of Kentucky, Lexington, Kentucky, U.S.A. (N.G.L.); Department of Statistics, University of Kentucky, Lexington, Kentucky, U.S.A. (G.S.H., K.L.T.); and Division of Athletic Training, West Virginia University, Morgantown, West Virginia, U.S.A. (K.N.J.).

The authors report the following potential conflicts of interest or sources of funding: Dr. Stone is a committee member of AANA and AOSSM. He has received nonfinancial support from Flexion Therapuetics, Allosource, and Smith and Nephew, as well as grants from Arthrex, personal fees from Medwest Associates, nonfinancial support from Wright Medical, outside of the submitted work. Dr. Jacobs reports grants and personal fees from Flexion Therapeutics and from Smith $\theta$ Nephew, outside the submitted work. Dr. of those aged 26-49 years reported having a major depressive episode in the past year. ${ }^{1}$ These numbers are even more striking in the hip arthroscopy population,

Duncan reports grants and personal fees from Smith and Nephew; grants from Stryker, Zimmer/Biomet, Bone Support, and Medtronic; and personal fees from OrthAlign, Heraeus, and Bone Support, outside of the submitted work. Full ICMJE author disclosure forms are available for this article online, as supplementary material.

Received December 2, 2020; accepted May 14, 2021.

Address correspondence to Anthony J. Zacharias, M.D., University of Kentucky, 740 S. Limestone, K401, Lexington, KY 40503 U.S.A. E-mail: Anthony.Zacharias@uky.edu

(C) 2021 THE AUTHORS. Published by Elsevier Inc. on behalf of the Arthroscopy Association of North America. This is an open access article under the CC BY-NC-ND license (http://creativecommons.org/licenses/by-nc-nd/4.0/).

2666-061X/201913

https://doi.org/10.1016/j.asmr.2021.05.005 
as mental health disorders have been reported in up to $45 \%$ of this patient population. ${ }^{2-4}$ Additionally, it has been reported that $48 \%$ of patients are taking at least 1 psychotropic medication at the time of hip arthroscopy. ${ }^{5}$ For patients undergoing hip arthroscopy, poor mental health has been correlated with worse preoperative patient-reported outcomes, ${ }^{3,4,6}$ persistent postoperative pain, ${ }^{7}$ and decreased postoperative patient reported outcomes (PROs). ${ }^{4,6,8-11}$

However, it remains unknown whether there is a potential bidirectional relationship between hip pain and poor mental health, as there is a paucity of research devoted to understanding how mental health disorders may be affected by surgical treatment of musculoskeletal conditions. Although not specific to those with preoperative depression or anxiety, Sochacki et al. demonstrated $66.2 \%$ of patients had minimal clinically important decreases in depressive symptoms following hip arthroscopy for femoral acetabular impingement syndrome (FAIS). ${ }^{6}$ With limited research on the topic, it remains unclear whether these decreased symptoms ${ }^{6}$ are also seen in patients undergoing hip arthroscopy with comorbid depression or anxiety that may be predisposed to an inferior outcome.

In the arthroplasty literature, the concept of medically optimizing patients before surgery to lessen the risk of complication and promote improved clinical outcomes has been widely adopted. ${ }^{12}$ Similar preoperative optimization is not widely adopted for hip arthroscopy. The lack of understanding regarding chronic hip pain, delayed presentation to the managing surgeon, ${ }^{13,14}$ and high use of mental health resources in the perioperative period further confounds the issue. ${ }^{15}$ The purpose of this study was to investigate whether patients undergoing arthroscopic treatment of femoral acetabular impingement syndrome (FAIS) decreased prescription treatment of comorbid depression and anxiety in the year following hip arthroscopy. Our hypothesis was that use of pharmacological treatment and psychological therapy for comorbid depression and anxiety would decrease in the year following hip arthroscopy secondary to FAIS.

\section{Methods}

Using the Truven Health Marketscan database and CPT codes 29914 (femoroplasty), 29915 (acetabuloplasty), and/or 29916 (labral repair), we identified 14,380 FAIS patients who underwent hip arthroscopy between January 2009 and December 2016. The Truven database contains Marketscan Commercial and Medicare Supplemental claims and encounters and includes more than 135 million unique individuals, which represents $20.3 \%$ of all non-CMS patients nationwide (CMS is Center for Medicare and Medicaid Services; Data source: Truven Health, Copyright 2012, 2017 Truven Health Analytics, Inc.).
Patients were included if they had one or more claims related to depression or anxiety during the year prior to surgery (ICD-9 codes 296, 298, 300, 309, and 311). Depression and anxiety diagnosis were used, as these have been previously shown to be correlated with preoperative and postoperative PROs. ${ }^{4,6,7,10,11}$ Patients were excluded if they had incomplete health insurance for 1 year either prior to or following surgery. Patients with a previous hip arthroscopy prior to the index procedure were excluded. For the subset of patients who underwent a subsequent hip arthroscopy after the index event, only the index event was included in the current analyses and not the secondary contralateral or revision procedure. For patients with depression and/or anxiety, claims relating to pharmaceutical and psychological therapy treatments for mental health disorders were identified. Four groups of patients were analyzed on the basis of preoperative anxiety/depression treatment: those who received medication only, therapy only, medication + therapy, and no treatment. The total days of medication received and number of therapy sessions for anxiety/depression treatment were recorded for the year prior to, and following, surgery. We also compared the number of patients in each group who filled a narcotic pain prescription within 180 days prior to surgery or $>90$ days after hip arthroscopy. This was done using National Drug Code (NDC) numbers associated with all opioid agonists; opioid partial agonists, such as tramadol; and opioid combination drug classes. ${ }^{16}$ Data on included patients has also been reported in a prior article. ${ }^{15}$

\section{Statistical Analysis}

Summary statistics were calculated from the Truven database to compare patient demographic variables across the four preoperative treatment groups described above. Because of the large sample sizes in this study population, traditional hypothesis testing and $P$ values are not appropriate to report, since even small differences between groups will appear to be highly significant despite not being clinically meaningful. ${ }^{17,18}$ However, paired $t$ tests were used to compare therapy visits and days of medication received for the small subset of the study population using these treatments during both the year prior to and following surgery. All analyses were completed using $\mathrm{R}$, version 3.6.1 (R Foundation for Statistical Computing; Vienna, Austria).

\section{Results}

Of the 14,830 patients that met the inclusion/exclusion criteria, 5,208 patients had a preoperative depression and/or anxiety claim prior to surgery $(35.1 \%)$. Preoperative treatment of depression and/or anxiety was as follows: medication only $(n=648,12.4 \%)$, therapy only $(n=899,17.3 \%)$, medication + therapy 
$(n=252,4.8 \%)$, and no treatment $(n=3,409,65.5 \%)$ (Table 1).

Nine hundred patients filled prescriptions for medications associated with depression or anxiety in the year prior to hip arthroscopy. The median number of days of medication prior to surgery was 120 days (interquartile range $=3$ to 330 ), and the median number of days of medication in the year after surgery was 90 days (interquartile range $=30$ to 270 ). Of the 900 patients originally prescribed anxiety/depressionrelated medication, $422(46.9 \%)$ did not fill an anxiety/depression-related prescription in the year following surgery. The mean change in total number of days of medication filled was a reduction of 66.1 days.

Psychotherapy was used by 1,151 patients in the year prior to hip arthroscopy. The median number of psychotherapy sessions prior to surgery was 6 sessions (interquartile range $=2$ to 12 ), and the median number psychotherapy sessions in the year after surgery was 5 sessions (interquartile range $=2$ to 11 ). Of the 1,151 patients receiving therapy related to anxiety/depression in the year prior to surgery, $549(47.7 \%)$ did not receive therapy in the year following surgery. Mean change in total number of psychotherapy sessions from the year prior to surgery to the year following surgery was a reduction of 2.1 sessions.

Preoperative opioid prescriptions were filled for 393 patients $(60.6 \%)$ in the medication-only group, 275 patients $(30.6 \%)$ in the therapy-only group, 156 patients $(61.9 \%)$ in the medication + therapy group, and 1,059 patients $(31.1 \%)$ in the no treatment group. All groups showed a reduction from preoperative to postoperative opioid use, defined as $>90$ days from surgery, with postoperative opioid use found in 330 patients $(50.9 \%)$ in the medication-only group, 225 patients $(25.0 \%)$ in the therapy-only group, 120 patients $(47.6 \%)$ in medication + therapy group, and 861 patients $(25.3 \%)$ in no-treatment group (Table 1).

\section{Discussion}

Results of this study support the hypothesis of decreased use of pharmacotherapy and psychotherapy treatments in patients that underwent hip arthroscopy with comorbid depression and/or anxiety. Both pharmacotherapy and psychotherapy treatment showed a similar reduction $(46.9 \%$ and $47.7 \%$, respectively) in claims in those patients receiving these treatments preoperatively. Although all groups reduced the number of opioid prescriptions, a secondary finding of this study was the increased proportion of patients in the medication-only and medication + therapy groups receiving preoperative and prolonged postoperative ( $>90$ days from surgery) opioids compared to therapyonly and no-treatment groups.

A strong body of literature has demonstrated the interaction of mental health on perception of pain and function prior to, and following, hip arthroscopy. ${ }^{3,4,6-11}$ Less studied is the impact of hip arthroscopy on psychological comorbidities. Following hip arthroscopy for FAIS, Sochacki et al. showed that $66.2 \%$ of patients had a minimal, clinically important difference in reduction scores from preoperative to 1 year postoperative on the Beck Depression Inventory-II. ${ }^{6}$ Chronic pain increases depressive symptoms, and with FAIS being a chronic condition, the removal of the pain generator via successful hip arthroscopy may then positively affect a patient's emotional well-being. However, we cannot assess causality as part of the insurance claims database analysis.

The results of the current study allude to a possible benefit following hip arthroscopy. The decreased use of depression/anxiety treatment-related medications and therapy should not be overlooked, as we assess developing strategies for comprehensive patient care. The next logical step is intervention-based strategies and the incorporation of preoperative treatment of comorbid psychological pathology prior to hip preservation. Richard et al. outlined their multidisciplinary approach toward adolescent hip preservation surgery. ${ }^{19}$ Although their study lacked a control group for comparison, the authors provided the framework on which future studies can build to improve clinical care.

\section{Link Between Psychotropic Medications and Both Preoperative and Postoperative Opioid Use}

Interestingly, for hip arthroscopy patients with comorbid depression or anxiety, opioid use both prior to and following surgery differs for the psychological treatments received. For context, in a previous study using the MarketScan database, $25.6 \%$ of patients filled

Table 1. Demographic Data

\begin{tabular}{|c|c|c|c|c|}
\hline Demographic Variable & Medication Only & Therapy Only & Medication + Therapy & Neither \\
\hline$\overline{\text { Patients }}$ & $648(12.4 \%)$ & $899(17.3 \%)$ & $252(4.8 \%)$ & $3,409(65.5 \%)$ \\
\hline Age (years) & $39.6 \pm 11.3$ & $32.3 \pm 13.5$ & $37.3 \pm 12.0$ & $34.9 \pm 13.1$ \\
\hline Female sex & $478(73.8 \%)$ & $658(73.2 \%)$ & $186(73.8 \%)$ & $2387(70.0 \%)$ \\
\hline Post-op opioid 90 days & $330(50.9 \%)$ & $225(25.0 \%)$ & $120(47.6 \%)$ & $861(25.3 \%)$ \\
\hline
\end{tabular}

Qualitative variables are reported using counts and within-group prevalence rates, while age was reported using within-group means \pm SD.

*Within 180 days of surgery. 
opioid prescriptions in the 180 days prior to surgery, and $23.4 \%$ filled opioid prescriptions $>90$ days after hip arthroscopy. ${ }^{15}$ In the current study, the prevalence of both preoperative and postoperative opioid use in the therapy only or no anxiety/depression treatment groups was very similar to those without comorbid psychological diagnoses (approximately 30\% preoperative and $25 \%$ postoperatively). On the contrary, the current study identified a potential link between psychotropic medications and both preoperative and postoperative opioid use. The prevalence of opioid use for those taking psychotropic medications was nearly double that of the therapy only or no anxiety/depression treatment groups both prior to (approximately $60 \%$ vs. $30 \%$ ) and following surgery (approximately $50 \%$ vs. $25 \%$ ).

While not limited to hip arthroscopy, preoperative psychotropic medication use has been associated with an increased risk of chronic opioid use after both lowrisk and major surgery. ${ }^{20,21}$ Antidepressants appear to carry the greatest risk, with studies reporting as high as a 14-fold increase in chronic postoperative opioid use when compared to the general population. ${ }^{22}$ The rationale for the increased prevalence of preoperative and postoperative opioid use for those taking psychotropic medications could be related to the severity of depression/anxiety, warranting medical treatment as opposed to therapy only and no treatment; however, analysis of multicollinearity could not be assessed in the current claims database analysis since symptom severity could not be determined. However, with previous reports linking psychotropic medication use with chronic postoperative opioid use, additional research is needed to elucidate the relationship and potential methods to reduce the risk for this apparent high-risk patient population.

\section{Limitations}

The current study is not without limitation. Any analysis of a claims-based database inherently relies on the accuracy of the coding. Disadvantages of using these data include incomplete, inaccurate, or missing data, as well as the appropriateness of treatment in some scenarios. The Truven database may not be generalizable to the U.S. population, as data are obtained from typically working-age patients covered by private insurance. In addition, larger employers typically contribute to this database compared to small- or medium-sized firms. ${ }^{23}$ Additionally, we were unable to determine whether depression/anxiety medications were used for treatment of musculoskeletal pain or specifically targeting the coded mental illness. We are unable to assess whether extenuating circumstances resulting in the absence to psychological therapy sessions, such as missing psychological therapy sessions in favor of physical therapy treatments. Other confounders, such as tobacco use were also not assessed in this study, which has previously been shown to effect results of hip arthroscopy, as well as correlate with mental health. ${ }^{24,25}$ Finally, without analysis of outcomes, such as pain scales and mental health scores, we were unable to assess for collinearity that may be present.

\section{Conclusion}

Hip arthroscopy for FAIS is associated with a decreased postoperative use of health care resources for the treatment of depression and anxiety. Clinicians should also be aware of the potential interplay between preoperative psychotropic medication use and prolonged opioid use when counseling patients.

\section{References}

1. Substance Abuse and Mental Health Services Administration. 2019 National Survey of Drug Use and Health (NSDUH) Releases. https://www.samhsa.gov/data/ release/2019-national-survey-drug-use-and-health-nsduhreleases. Published 2020. Accessed 10/16, 2020.

2. Rosenblum A, Landy DC, Perrone MA, Whyte N, Kang R. The presence of a psychiatric condition is associated with undergoing hip arthroscopy for femoroacetabular impingement: A matched case-controlled study. J Arthroplasty 2019;34:446-449.

3. Jacobs CA, Burnham JM, Jochimsen KN, Molina DT, Hamilton DA, Duncan ST. Preoperative symptoms in femoroacetabular impingement patients are more related TO mental health scores than the severity of labral tear or magnitude off bony deformity. J Arthroplasty 2017;32: 3603-3606.

4. Martin RL, Christoforetti JJ, McGovern R, et al. The impact of depression on patient outcomes in hip arthroscopic surgery. Orthop J Sports Med 2018;6: 2325967118806490.

5. Youlo ST, Walczak BE, Keene JS. Does the use of psychotropic medication adversely affect the outcomes of hip arthroscopy? Am J Sports Med 2018;46:3423-3428.

6. Sochacki KR, Brown L, Cenkus K, Di Stasi S, Harris JD, Ellis TJ. Preoperative depression is negatively associated with function and predicts poorer outcomes after hip arthroscopy for femoroacetabular impingement. Arthroscopy 2018;34:2368-2374.

7. Stone AV, Malloy P, Beck EC, et al. Predictors of persistent postoperative pain at minimum 2 years after arthroscopic treatment of femoroacetabular impingement. Am J Sports Med 2019;47:552-559.

8. Stephan P, Röling MA, Mathijssen NMC, Hannink G, Bloem RM. Developing a risk prediction model for the functional outcome after hip arthroscopy. BMC Musculoskelet Disord 2018;19:122.

9. Ernat JJ, Song DJ, Brugman SC, Shaha SH, Tokish JM, Lee GY. Mental health medication use correlates with poor outcome after femoroacetabular impingement surgery in a military population. J Bone Joint Surg Am 2015;97:1272-1277.

10. Nwachukwu BU, Beck EC, Lee EK, et al. Application of machine learning for predicting clinically meaningful 
outcome after arthroscopic femoroacetabular impingement surgery. Am J Sports Med 2020;48:415-423.

11. Stone AV, Beck EC, Malloy P, et al. Preoperative predictors of achieving clinically significant athletic functional status after hip arthroscopy for femoroacetabular impingement at minimum 2-year follow-up. Arthroscopy 2019;35:3049-3056.e3041.

12. Kim KY, Anoushiravani AA, Chen KK, et al. Perioperative orthopedic surgical home: optimizing total joint arthroplasty candidates and preventing readmission. J Arthroplasty 2019;34:S91-S96.

13. Kahlenberg CA, Han B, Patel RM, Deshmane PP, Terry MA. Time and cost of diagnosis for symptomatic femoroacetabular impingement. Orthop J Sports Med 2014;2:2325967114523916.

14. Petrera M, Yanez-Siller F, Whelan D, et al. Analysis of the referral pattern and wait time for hip arthroscopy in a single payer publicly funded health care system. J Eval Clin Pract 2020;26:81-85.

15. Jacobs CA, Hawk GS, Jochimsen KN, et al. Depression and anxiety are associated with increased health care costs and opioid use for patients with femoroacetabular impingement undergoing hip arthroscopy: Analysis of a claims database. Arthroscopy 2020;36:745-750.

16. Hankosky ER, Bush HM, Dwoskin LP, et al. Retrospective analysis of health claims to evaluate pharmacotherapies with potential for repurposing: Association of bupropion and stimulant use disorder remission. AMIA Annu Symp Proc 2018;2018:1292-1299.

17. Khalilzadeh J, Tasci AD. Large sample size, significance level, and the effect size: Solutions to perils of using big data for academic research. Tourism Management 2017;62:89-96.
18. Lantz B. The large sample size fallacy. Scand J Caring Sci 2013;27:487-492.

19. Richard HM, Nguyen DC, Podeszwa DA, De La Rocha A, Sucato DJ. Perioperative interdisciplinary intervention contributes to improved outcomes of adolescents treated with hip preservation surgery. J Pediatr Orthop 2018;38: 254-259.

20. Clarke H, Soneji N, Ko DT, Yun L, Wijeysundera DN. Rates and risk factors for prolonged opioid use after major surgery: population-based cohort study. BMJ 2014;348: g1251.

21. Alam A, Gomes T, Zheng H, Mamdani MM, Juurlink DN, Bell CM. Long-term analgesic use after low-risk surgery: a retrospective cohort study. Arch Intern Med 2012;172: 425-430.

22. Sun EC, Darnall BD, Baker LC, Mackey S. Incidence of and risk factors for chronic opioid use among opioid-naive patients in the postoperative period. JAMA Intern Med 2016;176:1286-1293.

23. Kulaylat AS, Schaefer EW, Messaris E, Hollenbeak CS. Truven health analytics MarketScan databases for clinical research in colon and rectal surgery. Clin Colon Rectal Surg 2019;32:54-60.

24. Lall AC, Hammarstedt JE, Gupta AG, et al. Effect of cigarette smoking on patient-reported outcomes in hip arthroscopic surgery: A matched-pair controlled study with a minimum 2-year follow-up. Orthop J Sports Med 2019;7:2325967118822837.

25. Fluharty M, Taylor AE, Grabski M, Munafò MR. The association of cigarette smoking with depression and anxiety: a systematic review. Nicotine Tob Res 2017;19: 3-13. 